\title{
Small Inductive Dimension of Topological Spaces. Part II
}

\author{
Karol Pąk \\ Institute of Computer Science \\ University of Białystok \\ Poland
}

\begin{abstract}
Summary. In this paper we present basic properties of $n$-dimensional topological spaces according to the book [10]. In the article the formalization of Section 1.5 is completed.
\end{abstract}

MML identifier: $\underline{\text { TOPDIM_2 }}$, version: $\underline{7.11 .04 \quad 4.130 .1076}$

The papers [15], [1], [3], [9], [5], [8], [16], [2], [4], [6], [13], [12], [17], [14], [18], [7], and [11] provide the terminology and notation for this paper.

\section{Order of a Family of Subsets of a Set}

In this paper $n$ denotes a natural number, $X$ denotes a set, and $F_{1}, G_{1}$ denote families of subsets of $X$.

Let us consider $X, F_{1}$. We say that $F_{1}$ is finite-order if and only if:

(Def. 1) There exists $n$ such that for every $G_{1}$ such that $G_{1} \subseteq F_{1}$ and $n \in \operatorname{Card} G_{1}$ holds $\cap G_{1}$ is empty.

Let us consider $X$. Observe that there exists a family of subsets of $X$ which is finite-order and every family of subsets of $X$ which is finite is also finite-order.

Let us consider $X, F_{1}$. The functor order $F_{1}$ yielding an extended real number is defined as follows:

(Def. 2)(i) For every $G_{1}$ such that order $F_{1}+1 \in \operatorname{Card} G_{1}$ and $G_{1} \subseteq F_{1}$ holds $\cap G_{1}$ is empty and there exists $G_{1}$ such that $G_{1} \subseteq F_{1}$ but Card $G_{1}=$ order $F_{1}+1$ but $\bigcap G_{1}$ is non empty or $G_{1}$ is empty if $F_{1}$ is finite-order,

(ii) order $F_{1}=+\infty$, otherwise. 
Let us consider $X$ and let $F$ be a finite-order family of subsets of $X$. Observe that order $F+1$ is natural and order $F$ is integer.

Next we state three propositions:

(1) If order $F_{1} \leq n$, then $F_{1}$ is finite-order.

(2) If order $F_{1} \leq n$, then for every $G_{1}$ such that $G_{1} \subseteq F_{1}$ and $n+1 \in \operatorname{Card} G_{1}$ holds $\cap G_{1}$ is empty.

(3) If for every finite family $G$ of subsets of $X$ such that $G \subseteq F_{1}$ and $n+1<$ $\overline{\bar{G}}$ holds $\cap G$ is empty, then order $F_{1} \leq n$.

\section{Basic Properties of $n$-Dimensional Topological Spaces}

One can verify that there exists a topological space which is finite-ind, second-countable, and metrizable.

For simplicity, we adopt the following convention: $T_{1}$ is a metrizable topological space, $T_{2}, T_{3}$ are finite-ind second-countable metrizable topological spaces, $A, B, L, H$ are subsets of $T_{1}, U, W$ are open subsets of $T_{1}, p$ is a point of $T_{1}$, $F, G$ are finite families of subsets of $T_{1}$, and $I$ is an integer.

We now state several propositions:

(4) Let given $T_{1}$. Suppose that

(i) $\quad T_{1}$ is second-countable, and

(ii) there exists $F$ such that $F$ is closed, a cover of $T_{1}$, countable, and finite-ind and ind $F \leq n$.

Then $T_{1}$ is finite-ind and ind $T_{1} \leq n$.

(5) Let $A, B$ be finite-ind subsets of $T_{1}$. Suppose $A$ is closed and $T_{1} \uparrow(A \cup B)$ is second-countable and ind $A \leq I$ and ind $B \leq I$. Then $\operatorname{ind}(A \cup B) \leq I$ and $A \cup B$ is finite-ind.

(6) Let given $T_{1}$. Suppose $T_{1}$ is second-countable and finite-ind and ind $T_{1} \leq$ $n$. Then there exist $A, B$ such that $\Omega_{\left(T_{1}\right)}=A \cup B$ and $A$ misses $B$ and ind $A \leq n-1$ and ind $B \leq 0$.

(7) Let given $T_{1}$. Suppose $T_{1}$ is second-countable and finite-ind and ind $T_{1} \leq$ $I$. Then there exists $F$ such that

(i) $\quad F$ is a cover of $T_{1}$ and finite-ind,

(ii) ind $F \leq 0$,

(iii) $\overline{\bar{F}} \leq I+1$, and

(iv) for all $A, B$ such that $A, B \in F$ and $A$ meets $B$ holds $A=B$.

(8) Let given $T_{1}$. Suppose $T_{1}$ is second-countable and there exists $F$ such that $F$ is a cover of $T_{1}$ and finite-ind and ind $F \leq 0$ and $\overline{\bar{F}} \leq I+1$. Then $T_{1}$ is finite-ind and ind $T_{1} \leq I$.

Let $T_{1}$ be a second-countable metrizable topological space and let $A, B$ be finite-ind subsets of $T_{1}$. One can check that $A \cup B$ is finite-ind. 
Next we state two propositions:

(9) If $A$ is finite-ind and $B$ is finite-ind and $T_{1} \uparrow(A \cup B)$ is second-countable, then $A \cup B$ is finite-ind and $\operatorname{ind}(A \cup B) \leq \operatorname{ind} A+\operatorname{ind} B+1$.

(10) For all topological spaces $T_{4}, T_{5}$ and for every subset $A_{1}$ of $T_{4}$ and for every subset $A_{2}$ of $T_{5}$ holds $\operatorname{Fr}\left(A_{1} \times A_{2}\right)=\operatorname{Fr} A_{1} \times \overline{A_{2}} \cup \overline{A_{1}} \times \operatorname{Fr} A_{2}$.

Let us consider $T_{2}, T_{3}$. Observe that $T_{2} \times T_{3}$ is finite-ind.

We now state several propositions:

(11) Let given $A, B$. Suppose $A$ is closed and $B$ is closed and $A$ misses $B$. Let given $H$. Suppose ind $H \leq n$ and $T_{1}\lceil H$ is second-countable and finite-ind. Then there exists $L$ such that $L$ separates $A, B$ and $\operatorname{ind}(L \cap H) \leq n-1$.

(12) Let given $T_{1}$. Suppose $T_{1}$ is finite-ind and second-countable and ind $T_{1} \leq$ $n$. Let given $A, B$. Suppose $A$ is closed and $B$ is closed and $A$ misses $B$. Then there exists $L$ such that $L$ separates $A, B$ and ind $L \leq n-1$.

(13) Let given $H$. Suppose $T_{1} \uparrow H$ is second-countable. Then $H$ is finite-ind and ind $H \leq n$ if and only if for all $p, U$ such that $p \in U$ there exists $W$ such that $p \in W$ and $W \subseteq U$ and $H \cap \operatorname{Fr} W$ is finite-ind and $\operatorname{ind}(H \cap \operatorname{Fr} W) \leq$ $n-1$.

(14) Let given $H$. Suppose $T_{1} \uparrow H$ is second-countable. Then $H$ is finite-ind and ind $H \leq n$ if and only if there exists a basis $B_{1}$ of $T_{1}$ such that for every $A$ such that $A \in B_{1}$ holds $H \cap \operatorname{Fr} A$ is finite-ind and $\operatorname{ind}(H \cap \operatorname{Fr} A) \leq n-1$.

(15) If $T_{2}$ is non empty or $T_{3}$ is non empty, then $\operatorname{ind}\left(T_{2} \times T_{3}\right) \leq \operatorname{ind} T_{2}+\operatorname{ind} T_{3}$.

(16) If ind $T_{3}=0$, then $\operatorname{ind}\left(T_{2} \times T_{3}\right)=\operatorname{ind} T_{2}$.

\section{Small Inductive Dimension of Euclidean Spaces}

For simplicity, we follow the rules: $u$ denotes a point of $\mathcal{E}^{1}, U$ denotes a point of $\mathcal{E}_{\mathrm{T}}^{1}, r, u_{1}$ denote real numbers, and $s$ denotes a real number.

Next we state three propositions:

(17) If $\left\langle u_{1}\right\rangle=u$ and $r>0$, then $\overline{\operatorname{Ball}}(u, r)=\left\{\langle s\rangle: u_{1}-r \leq s \wedge s \leq u_{1}+r\right\}$.

(18) If $\left\langle u_{1}\right\rangle=U$ and $r>0$, then $\operatorname{Fr} \operatorname{Ball}(U, r)=\left\{\left\langle u_{1}-r\right\rangle,\left\langle u_{1}+r\right\rangle\right\}$.

(19) Let $T$ be a topological space and $A$ be a countable subset of $T$. If $T\lceil A$ is a $T_{4}$ space, then $A$ is finite-ind and ind $A \leq 0$.

Let $T_{1}$ be a metrizable topological space. Observe that every subset of $T_{1}$ which is countable is also finite-ind.

Let $n$ be a natural number. Observe that $\mathcal{E}_{\mathrm{T}}^{n}$ is finite-ind.

One can prove the following propositions:

(20) If $n \leq 1$, then $\operatorname{ind}\left(\mathcal{E}_{\mathrm{T}}^{n}\right)=n$.

(21) $\operatorname{ind}\left(\mathcal{E}_{\mathrm{T}}^{n}\right) \leq n$. 
(22) Let given $A$. Suppose $T_{1} \uparrow A$ is second-countable and finite-ind and ind $A \leq 0$. Let given $F$. Suppose $F$ is open and a cover of $A$. Then there exists a function $g$ from $F$ into $2^{\text {the carrier of } T_{1}}$ such that

(i) $\operatorname{rng} g$ is open,

(ii) $\operatorname{rng} g$ is a cover of $A$,

(iii) for every set $a$ such that $a \in F$ holds $g(a) \subseteq a$, and

(iv) for all sets $a, b$ such that $a, b \in F$ and $a \neq b$ holds $g(a)$ misses $g(b)$.

(23) Let given $T_{1}$. Suppose $T_{1}$ is second-countable and finite-ind and ind $T_{1} \leq$ $n$. Let given $F$. Suppose $F$ is open and a cover of $T_{1}$. Then there exists $G$ such that $G$ is open, a cover of $T_{1}$, and finer than $F$ and $\overline{\bar{G}} \leq \overline{\bar{F}} \cdot(n+1)$ and order $G \leq n$.

(24) Let given $T_{1}$. Suppose $T_{1}$ is finite-ind. Let given $A$. Suppose $\operatorname{ind}\left(A^{\mathrm{c}}\right) \leq n$ and $T_{1}\left\lceil A^{\mathrm{c}}\right.$ is second-countable. Let $A_{1}, A_{2}$ be closed subsets of $T_{1}$. Suppose $A=A_{1} \cup A_{2}$. Then there exist closed subsets $X_{1}, X_{2}$ of $T_{1}$ such that $\Omega_{\left(T_{1}\right)}=$ $X_{1} \cup X_{2}$ and $A_{1} \subseteq X_{1}$ and $A_{2} \subseteq X_{2}$ and $A_{1} \cap X_{2}=A_{1} \cap A_{2}=X_{1} \cap A_{2}$ and $\operatorname{ind}\left(X_{1} \cap X_{2} \backslash A_{1} \cap A_{2}\right) \leq n-1$.

\section{REFERENCES}

[1] Grzegorz Bancerek. Cardinal numbers. Formalized Mathematics, 1(2):377-382, 1990.

[2] Grzegorz Bancerek. The fundamental properties of natural numbers. Formalized Mathematics, 1(1):41-46, 1990.

[3] Grzegorz Bancerek. König's theorem. Formalized Mathematics, 1(3):589-593, 1990.

[4] Grzegorz Bancerek. The ordinal numbers. Formalized Mathematics, 1(1):91-96, 1990.

[5] Grzegorz Bancerek and Krzysztof Hryniewiecki. Segments of natural numbers and finite sequences. Formalized Mathematics, 1(1):107-114, 1990.

[6] Leszek Borys. Paracompact and metrizable spaces. Formalized Mathematics, 2(4):481485, 1991.

[7] Agata Darmochwał. Families of subsets, subspaces and mappings in topological spaces. Formalized Mathematics, 1(2):257-261, 1990.

[8] Agata Darmochwał. Finite sets. Formalized Mathematics, 1(1):165-167, 1990.

[9] Agata Darmochwał. The Euclidean space. Formalized Mathematics, 2(4):599-603, 1991.

[10] Ryszard Engelking. Teoria wymiaru. PWN, 1981.

[11] Robert Milewski. Bases of continuous lattices. Formalized Mathematics, 7(2):285-294, 1998.

[12] Beata Padlewska. Families of sets. Formalized Mathematics, 1(1):147-152, 1990.

[13] Beata Padlewska and Agata Darmochwał. Topological spaces and continuous functions. Formalized Mathematics, 1(1):223-230, 1990.

[14] Karol Pąk. Small inductive dimension of topological spaces. Formalized Mathematics, 17(3):207-212, 2009, doi: 10.2478/v10037-009-0025-7.

[15] Andrzej Trybulec. A Borsuk theorem on homotopy types. Formalized Mathematics, 2(4):535-545, 1991.

[16] Michał J. Trybulec. Integers. Formalized Mathematics, 1(3):501-505, 1990.

[17] Zinaida Trybulec. Properties of subsets. Formalized Mathematics, 1(1):67-71, 1990.

[18] Mirosław Wysocki and Agata Darmochwał. Subsets of topological spaces. Formalized Mathematics, 1(1):231-237, 1990.

Received August 7, 2009 